\title{
COMPARISON BETWEEN 2D TURBULENCE MODEL ESEL AND EXPERIMENTAL DATA FROM AUG AND COMPASS TOKAMAKS
}

\author{
Peter Ondáč $\check{C}^{a, b, *}$, JAn HoraceK ${ }^{b}, \mathrm{JAKUb} \mathrm{SeIdL}^{b}$, Petr VondráČeK $^{a, b}$, \\ Hans Werner MüLler ${ }^{c}$, Jiří AdámeK ${ }^{b}$, Anders Henry Nielsen ${ }^{d}$, \\ AND ASDEX UPGRADE TEAM ${ }^{c}$
}

\author{
${ }^{a}$ Department of Surface and Plasma Science, Faculty of Mathematics and Physics, Charles University, \\ $V$ Holešovičkách 2, 18000 Prague 8, Czech Republic \\ ${ }^{b}$ Institute of Plasma Physics, Academy of Sciences, Za Slovankou 1782/3, 18200 Prague 8, Czech Republic \\ ${ }^{c}$ Max-Planck-Institut für Plasmaphysik, Boltzmannstraße 2, D-85748 Garching, Germany \\ $d$ Association Euratom-Risø National Laboratory, Technical University of Denmark, OPL-128 Risø, Roskilde, \\ DK-4000, Denmark \\ * corresponding author: shreder.peter.ondac@gmail.com
}

\begin{abstract}
In this article we have used the 2D fluid turbulence numerical model, ESEL, to simulate turbulent transport in edge tokamak plasma. Basic plasma parameters from the ASDEX Upgrade and COMPASS tokamaks are used as input for the model, and the output is compared with experimental observations obtained by reciprocating probe measurements from the two machines. Agreements were found in radial profiles of mean plasma potential and temperature, and in a level of density fluctuations. Disagreements, however, were found in the level of plasma potential and temperature fluctuations. This implicates a need for an extension of the ESEL model from 2D to 3D to fully resolve the parallel dynamics, and the coupling from the plasma to the sheath.
\end{abstract}

KEYWORDS: turbulence; tokamak; computer model; probe measurements.

\section{INTRODUCTION}

Transport (mainly turbulent) in the outermost plasma region, in contact with material surfaces, regulates particle and heat loads on plasma-facing components. The control of this transport is very important for particle and heat confinements in tokamaks and other magnetized plasma experiments (stellarators, linear devices, reversed field pinches). Only intermittent turbulent structures account for more than $50 \%$ [1] of the radial plasma transport towards the material surfaces. Cross-field particles and heat losses from the central plasma represent a very high risk of damaging the tokamak first wall, and other plasma-facing components. At the same time, impurities released by these components spread through the boundary region into the central plasma, cooling it down by their radiation and decreasing the fusion rate due to the dilution of the fuel.

Interchange instability is one of the candidates for explaining these significant cross-field plasma losses observed at the tokamak edge. The numerical model ESEL (Edge-Sol-ELectrostatic) 224 simulates the edge turbulent plasma, as three interacting fluid fields, electron temperature, density and vorticity. In this article, following [5], we compare the predictions of the model with experimental measurements on two tokamaks with ITER-like geometry, ASDEX Upgrade [6] and COMPASS [7]. In the past, similar comparisons were made also for tokamaks JET [8], TCV [3], TEXTOR [9] and MAST [10. From 8 it follows that the radial transport in edge plasma is dominated by electrostatic interchange turbulence. Initially ESEL was used for TCV plasma with high collisionality, and was very successful. Quantities calculated from the measured density were in good concurrence with the model [3], [11 13. However, in comparisons between the ESEL and the ASDEX Upgrade plasma, with higher temperature and therefore lower collisionality than in TCV tokamak, there were great discrepancies [5]. In this case the temperature and potential were measured as well. The radial profile of the density in the ESEL was too flat, and relative temperature and electric potential fluctuations in the model were too large compared with experimental values. The radial profile of electric potential also differed in the model and the experiment. Later, a new extra linear term (described below) was added into one ESEL governing equation. With this term the model electric potential radial profile was finally in agreement with the ASDEX data, and the radial profile of density was more consistent with the ASDEX data. However the relative density, temperature and electric potential fluctuations were even larger than before [5]. From this time forth, a change in the extra term and extensive investigation of the parameter dependence in ESEL matching the ASDEX Upgrade plasma has been made. Also, first time comparisons between ESEL and COMPASS tokamaks have been made. A brief summary of the results is presented in this paper. In this context, the purpose of this work is to show 


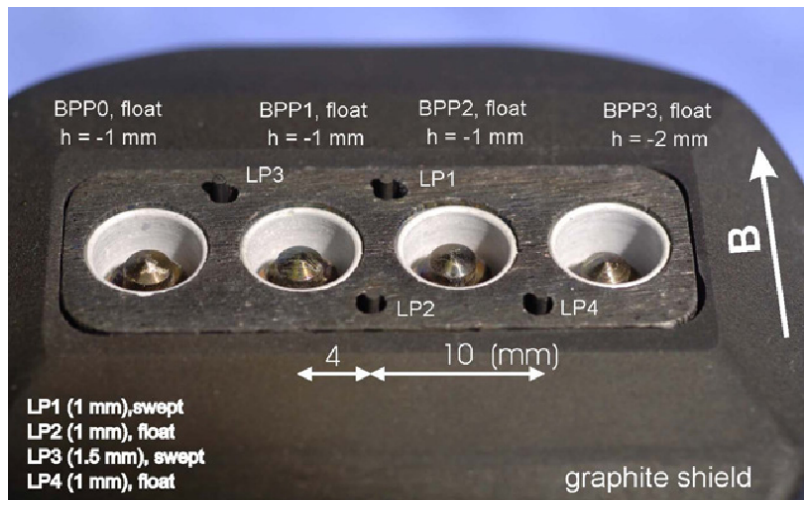

Figure 1. A photograph of the probe head with 4 Langmuir probes (LPs) and 4 ball-pen probes (BPPs) used in the ASDEX Upgrade. The vertical axis of the image corresponds to the direction parallel to $\boldsymbol{B}$ and the horizontal axis corresponds to the poloidal direction in the tokamak 16 .

the actual possibilities of the interpretations of the experimental data with the help of the interchange turbulence paradigm in the 2D model ESEL.

As far as the experiment is concerned, to measure individual turbulent structures (blobs) a high temporal (1 $\mathrm{\mu s})$ and spatial $(2 \mathrm{~mm})$ resolution was necessary. This was provided by a set of Langmuir probes (LPs) and ball-pen probes (BPPs) [14] mounted on a reciprocating manipulator.

The description of the experimental setup and the ESEL model is given in chapters 2 and 3. In chapter 4 we present the results obtained from the comparison between the experiment and model. A detailed study of the comparison can be found in the master thesis 15 .

\section{EXPERIMENTS}

The data from the ASDEX Upgrade tokamak shown in this article comes from discharge \#24349. They were previously analysed in [5] and [16], here, however, we use more general assumptions such as ratio of ion and electron temperature larger than one. The experiment was performed using the reciprocating horizontal manipulator located just above $(z=0.3125$ $\mathrm{m})$ the LFS mid-plane in the SOL [16]. The probe head used is shown in Figure 1.

The diameter of the ball-pen [14] collectors was $4 \mathrm{~mm}$ and the interior diameter of the shielding tubes was $6 \mathrm{~mm}$. The diameter of LP pins was $0.9 \mathrm{~mm}$. The BPPs and LPs were located in different poloidals and due to the poloidal inclination of the magnetic flux surface with respect to the probe head surface $\approx 12^{\circ}$, were also in different radial positions. In this work we use data from probes LP1, LP2, BPP1 and BPP2. The measurements were performed in a Dshaped deuterium plasma, during an L-mode with a neutral beam injection power of $\approx 1 \mathrm{MW}$ and a plasma current of $I_{\mathrm{p}} \approx 800 \mathrm{kA}$, line averaged density $n_{\mathrm{e}} \approx 3$. $10^{19} \mathrm{~m}^{-3}$ and toroidal magnetic field $B_{\mathrm{T}} \approx 2.5 \mathrm{~T}$ in the plasma centre and $\approx 1.9 \mathrm{~T}$ in the SOL. The sampling frequency of the data acquisition system was $2 \mathrm{MHz}$. For the purpose of statistical analysis, the radial probe head movement was divided into 60 time intervals, and each processed separately. These analysed time intervals, from which the experimental data points (further marked with circles) were obtained, had a duration of $2.9 \mathrm{~ms}$ and each of them corresponded to 5826 measured values. Each time interval corresponds to a corresponding radial position where the probes, during the interval, were situated. In the figures, the experimental data from both radial motion in and out of the plasma are shown (two lines with circles in each graph for ASDEX Upgrade labelled as "ASDEX", the smaller values are related to slower motion out). The probes BPP1, BPP2 and LP2 were floating and they measured the floating potentials $V_{\mathrm{f}}^{\mathrm{BPP} 1}, V_{\mathrm{f}}^{\mathrm{BPP} 2}$ and $V_{\mathrm{f}}^{\mathrm{LP} 2}$ respectively. LP1 was biased and measured ion saturation current, $I_{\text {is }}$. Plasma potential $\phi_{p}$ (in V), electron temperature $T_{\mathrm{e}}$ (in $\mathrm{eV}$ ) and plasma density $n_{\mathrm{e}}$ at the position of LP1 (in $\mathrm{m}^{-3}$ ) were calculated as following $\left(V_{\mathrm{f}}^{\mathrm{BPP}} \approx \phi_{\mathrm{p}}-0.6\left(T_{\mathrm{e}} / e\right)\right.$, the coefficient 0.6 has been found experimentally [17]):

$$
\begin{aligned}
\phi_{\mathrm{p}} & =\frac{V_{\mathrm{f}}^{\mathrm{BPP} 1}+V_{\mathrm{f}}^{\mathrm{BPP} 2}}{2}, \\
T_{\mathrm{e}} & =\frac{T_{\mathrm{e}}^{\mathrm{BPP} 1-\mathrm{LP} 2}+T_{\mathrm{e}}^{\mathrm{BPP} 2-\mathrm{LP} 2}}{2}, \\
T_{\mathrm{e}}^{\mathrm{BPP} 1-\mathrm{LP} 2} & =\frac{\left(V_{\mathrm{f}}^{\mathrm{BPP} 1}-V_{\mathrm{f}}^{\mathrm{LP} 2}\right) e}{\alpha-0.6}, \\
T_{\mathrm{e}}^{\mathrm{BPP} 2-\mathrm{LP} 2} & =\frac{\left(V_{\mathrm{f}}^{\mathrm{BPP} 2}-V_{\mathrm{f}}^{\mathrm{LP} 2}\right) e}{\alpha-0.6}, \\
n_{\mathrm{e}}=n_{\mathrm{e}}^{\mathrm{LP} 1} & =\frac{13 \cdot 10^{18} \mathrm{~m}^{-3}(\mathrm{eV})^{1 / 2} \sqrt{2}}{0.014 \mathrm{~A}} \frac{I_{i s}}{\sqrt{T_{e}+T_{\mathrm{i}}}},
\end{aligned}
$$

where coefficient $\alpha$ indicates representation of temperature fluctuations in the measured $V_{\mathrm{f}}^{\mathrm{LP} 2}$ :

$$
\alpha=-\frac{1}{2} \ln \left[2 \pi\left(\frac{m_{\mathrm{e}}}{m_{\mathrm{i}}}\right)\left(1+\frac{T_{\mathrm{i}}}{T_{\mathrm{e}}}\right)\right],
$$

with the electron and ion mass $m_{\mathrm{e}}$ and $m_{\mathrm{i}}$ and the electron and ion temperature $T_{\mathrm{e}}$ and $T_{\mathrm{i}}$. Secondary electron emission from the probes is neglected. In [16] only the value $\alpha \approx 2.8$, for $T_{\mathrm{i}} / T_{\mathrm{e}}=1$ has been used. Since the ratio $T_{\mathrm{i}} / T_{\mathrm{e}}$ can be up to 10 ([18], [20]), the corresponding $2<\alpha<2.8$.

In equation $(3)$, the constant $13 \cdot 10^{18} \mathrm{~m}^{-3}(\mathrm{eV})^{1 / 2}$ $/ 0.014 \mathrm{~A}$ is a calibration factor to match the lithium beam diagnostic measurement.

The experimental data from the COMPASS tokamak was obtained in discharge \#6092. The probe head shown in Figure 2 is a bit smaller and so less perturbing to the plasma. It was radially inserted into the plasma by the fast reciprocating horizontal mid-plane manipulator that is located at the outer mid-plane $(z=0)$. 


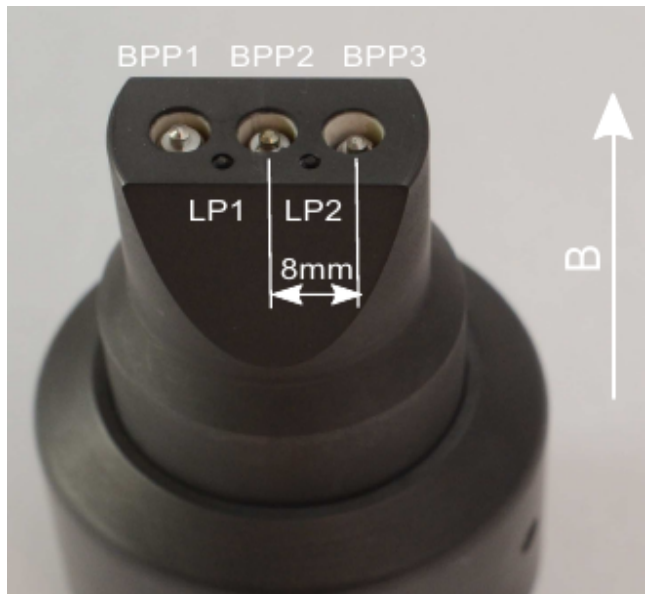

Figure 2. Photograph of the COMPASS probe head with 2 Langmuir probes (LPs) and 3 ball-pen probes (BPPs). The vertical and horizontal direction of the image parallel to the probe head surface corresponds to the toroidal and poloidal direction respectively [18.

The ball-pen probes had collectors with diameters of $2 \mathrm{~mm}$ and shielding with interior diameter of $5 \mathrm{~mm}$. LP pins had a diameter of $0.9 \mathrm{~mm}$ and protruded 1.5 $\mathrm{mm}$ into the plasma. The poloidal distance between LPs and BPPs was around $4 \mathrm{~mm}$. The measurements were performed in a D-shaped plasma with ohmic heating in L-mode with typical values of toroidal magnetic field $B_{\mathrm{T}}=1.16 \mathrm{~T}$ (at the minor axis at $R \approx 0.56 \mathrm{~m}$ ), plasma current $I_{\mathrm{p}}=110 \mathrm{kA}$ and line averaged electron density $n_{\mathrm{e}} \approx 5 \cdot 10^{19} \mathrm{~m}^{-3}$. The sampling frequency of the data acquisition system was $5 \mathrm{MHz}$. Analysed time intervals had durations of approximately $2 \mathrm{~ms}$, a compromise between long enough statistics (we typically caught 10 blobs during this interval) and short enough probe movement $(2 \mathrm{~mm}$, i.e., much less than typical blob size or the SOL radial decay length). Similar to the data from ASDEX Upgrade, we derive $n_{\mathrm{e}}$, $T_{\mathrm{e}}$ and $\phi_{\mathrm{p}}$ from the probe data, and for each time interval we calculate the first two statistical moments. The relative fluctuations were calculated as a ratio between the second and the first statistical moment. In the case of COMPASS measurements, we used only the data corresponding to the probe motion into the plasma - one experimental line with circles. Movement out of the plasma was not processed due to the arcing of the probes.

The probes BPP2 and LP2 were floating and measured floating potential $V_{\mathrm{f}}^{\mathrm{BPP} 2}$ and $V_{\mathrm{f}}^{\mathrm{LP} 2}$ respectively. LP1 was biased and measured ion saturation current $I_{\text {is }}$. The $T_{\mathrm{e}}($ in $\mathrm{eV}), \phi_{\mathrm{p}}($ in $\mathrm{V})$ and $n_{\mathrm{e}}\left(\right.$ in $\left.\mathrm{m}^{-3}\right)$ were calculated as following:

$$
\begin{gathered}
T_{\mathrm{e}} \simeq T_{\mathrm{e}}^{\mathrm{BPP} 2-\mathrm{LP} 2}=\frac{\left(V_{\mathrm{f}}^{\mathrm{BPP} 2}-V_{\mathrm{f}}^{\mathrm{LP} 2}\right) e}{\alpha-0.6}, \\
\phi_{\mathrm{p}}=V_{\mathrm{f}}^{\mathrm{BPP} 2}+0.6 \frac{T_{\mathrm{e}}}{e} \\
n_{\mathrm{e}}=n_{\mathrm{e}}^{\mathrm{LP} 1}=\left(2 \frac{\sqrt{m_{\mathrm{i}}}}{e S}\right) \frac{I_{\mathrm{is}}}{\sqrt{T_{\mathrm{e}}+T_{\mathrm{i}}}},
\end{gathered}
$$

where $S$ is a Langmuir probe effective collection area.

Since ion temperature $T_{\mathrm{i}}$ was not measured, we assumed for both tokamaks its value to be in the range of 1 to 10 times the electron temperature, in agreement with [19] or [20]. This uncertainty is represented by error bars in all figures with radial profiles of mean plasma density and electron temperature. In all ESEL simulations the ion temperature was set twice the electron temperature.

\section{ModeL ESEL}

The ESEL model (Edge-Sol ELectrostatic) is a 2D drift-fluid turbulence numerical simulation of scrapeoff layer turbulence. It is an electrostatic model (so the magnetic field is constant in time). There are three governing equations describing the evolution of plasma; electron density $n$, electron temperature $T_{\mathrm{e}}$ and electric potential $\phi$, which are solved in slab geometry perpendicular to the magnetic field (axis $x^{\prime}$, $\left.y^{\prime}, z^{\prime}\right)$ and which, by using the Bohm normalization (dimensionless quantities are marked with prime "'), take the following form [21]:

$$
\begin{aligned}
& \frac{\mathrm{d} n^{\prime}}{\mathrm{d} t^{\prime}}+n^{\prime} C^{\prime}\left(\phi^{\prime}\right)-C^{\prime}\left(n^{\prime} T_{\mathrm{e}}^{\prime}\right)=D_{\perp \mathrm{n}}^{\prime} \nabla_{\perp}^{\prime 2} n^{\prime}-\frac{n^{\prime}}{\tau_{\| n}^{\prime}}, \\
& \frac{\mathrm{d} T_{\mathrm{e}}^{\prime}}{\mathrm{d} t^{\prime}}+\frac{2}{3} T_{\mathrm{e}}^{\prime} C^{\prime}\left(\phi^{\prime}\right)-\frac{7}{3} T_{\mathrm{e}}^{\prime} C^{\prime}\left(T_{\mathrm{e}}^{\prime}\right) \\
& -\frac{2}{3} \frac{T_{\mathrm{e}}^{\prime 2}}{n^{\prime}} C^{\prime}\left(n^{\prime}\right)=D_{\perp T_{\mathrm{e}}}^{\prime} \nabla_{\perp}^{\prime 2} T_{\mathrm{e}}^{\prime}-\frac{T_{\mathrm{e}}^{\prime}}{\tau_{\| T_{\mathrm{e}}}^{\prime}}, \\
& \frac{\mathrm{d} \Omega^{\prime}}{\mathrm{d} t^{\prime}}-C^{\prime}\left(n^{\prime} T_{\mathrm{e}}^{\prime}\right)=D_{\perp \Omega}^{\prime} \nabla_{\perp}^{\prime 2} \Omega^{\prime}-\frac{\Omega^{\prime}}{\tau_{\| \Omega}^{\prime}} \\
& +\frac{2 c_{\mathrm{s}}}{\omega_{\mathrm{ci0} 0} L_{\| \mathrm{d}}} \Psi \\
& \Psi=\left[1-\exp \left(\alpha-\frac{\phi^{\prime}}{T_{\mathrm{e}}^{\prime}}\right)\right],
\end{aligned}
$$

where $\omega_{\text {ci0 }}=e B_{0} / m_{\mathrm{i}}$ is the ion gyro-frequency, $c_{\mathrm{s}}$ is the warm $\left(T_{\mathrm{i}} \neq 0\right)$ ion plasma sound speed and $L_{\| \mathrm{d}}$ is the parallel connection length for SOL between two divertors. The vorticity, the advective derivative, the inhomogeneous magnetic field and the curvature operator are defined by

$$
\begin{aligned}
\Omega^{\prime} & =\nabla_{\perp}^{\prime 2} \phi^{\prime}, & \frac{\mathrm{d}}{\mathrm{d} t^{\prime}} & =\frac{\partial}{\partial t^{\prime}}+\frac{1}{B^{\prime}} \boldsymbol{z} \times \nabla^{\prime} \phi^{\prime} \cdot \nabla^{\prime} \\
\frac{1}{B^{\prime}} & =1+\frac{r_{0}+\rho_{\mathrm{s} 0} x^{\prime}}{R_{0}}, & C^{\prime} & =-\frac{\rho_{\mathrm{s} 0}}{R_{0}} \frac{\partial}{\partial y^{\prime}},
\end{aligned}
$$

with the hybrid thermal gyro-radius $\rho_{\mathrm{s} 0}=c_{\mathrm{s} 0} / \omega_{\mathrm{ci} 0}$, and the tokamak major and minor radius $R_{0}$ and $r_{0}$. Here $c_{\mathrm{s} 0}=\sqrt{\left(T_{\mathrm{e} 0} / m_{\mathrm{i}}\right)}$ is the cold ion plasma sound speed, $T_{\mathrm{e} 0}$ is the electron temperature at the LCFS and $B_{0}$ is the magnetic field at the plasma centre.

For the purpose of this paper, we added the last term in the equation 9 into the ESEL model, in order to better match the experimental data - see later. This last term has, in dimensional form, the meaning of the average of the divergence of the parallel electric current 
to the divertor plasma sheath multiplied by $B /\left(n m_{\mathrm{i}}\right)$. The average is taken over the parallel direction with a connection length $L_{\| \mathrm{d}}$. Thus we have [22]:

$$
\begin{aligned}
\left\langle\nabla \cdot \boldsymbol{J}_{\|}\right\rangle & \approx\left\langle\boldsymbol{b} \cdot \nabla J_{\|}\right\rangle \\
& =\left\langle\nabla\left(\operatorname{enc}_{\mathrm{s}}\left[1-\exp \left(e V_{\mathrm{fd}} / T_{\mathrm{ed}}\right)\right]\right)\right\rangle \\
& \approx\left(2 \mathrm{enc}_{s} / L_{\| \mathrm{d}}\right)\left[1-\exp \left(-e V_{\mathrm{fd}} / T_{e}\right)\right]
\end{aligned}
$$

where $\boldsymbol{b}$ is the unit vector along the magnetic field, $T_{\text {ed }} \approx T_{\mathrm{e}}$ is the electron temperature in the vicinity of the divertor and $-V_{\mathrm{fd}} \approx \phi-\alpha\left(T_{\mathrm{e}} / e\right)$ is the floating potential at the divertor. This means that the term describes the outflow of electric potential from the blobs in parallel direction to what affects the radial turbulent transport (via $\boldsymbol{E} \times \boldsymbol{B}$ drift). The parallel terms are apparently the weakest point of the ESEL and there has been a lot of effort to develop a more sophisticated model of parallel transport. A similar sheath dissipation term has already been used before, in other 2D interchange codes, [23] or [24], but in ESEL, it was used the first time.

The parallel transport along the magnetic field lines is estimated in the model by characteristic loss times for particles $\left(\tau_{\| \mathrm{n}}^{\prime}\right)$, electron heat $\left(\tau_{\| \mathrm{T}_{\mathrm{e}}}^{\prime}\right)$ and momentum $\left(\tau_{\| \Omega}^{\prime}\right)$. Perpendicular diffusion due to collisions is represented by neoclassical Pfirsch-Schlüter perpendicular collisional diffusion coefficients for particles $D_{\perp \mathrm{n}}^{\prime}$, electron heat $\left(D_{\perp \mathrm{T}_{\mathrm{e}}}^{\prime}\right)$ and momentum $\left(D_{\perp \Omega}^{\prime}\right)$. The relations for computations of the loss times and the diffusion coefficients are shown in 21 .

The parallel particle density loss time in ESEL is estimated as $\tau_{\| \mathrm{n}} \simeq L_{\| \mathrm{m}} / v_{\|}$, where $v_{\|}$is parallel velocity equal around half $( \pm 30 \%)$ the warm ion sound speed. It is derived for time-independent parallel transport and it neglects the recycling of neutrals in the vicinity of the divertor as the particle source to the computational domain area. It would be more correct to use the length scale of parallel particle density variation $L_{\| \mathrm{n}}$ in place of $L_{\| \mathrm{m}}$, but it is difficult to estimate this 21.

These three governing equations $7,8,90$ were derived from the equation of continuity, fluid equation of motion and energy transport equation [15], [25]. Input data for the ESEL model are mainly electron temperature, electric potential and electron density at the separatrix or on the left edge (in the figures labelled as "LE") of the 2D computational domain, also a value of magnetic field in the plasma centre, tokamak geometry parameters and a selection of radial Neumann or Dirichlet boundary conditions. In poloidal direction there are only periodic boundary conditions. The model then provides a time series of turbulent fluctuations at predefined spatial points. Radial profiles and relative fluctuations are the model's output. Finite Larmor radius and in some cases also ion temperature are neglected. The computational domain at the outboard midplane of the tokamak is shown in Figure 3. The EDGE, SOL and WALL SHADOW region represent the confined plasma, SOL

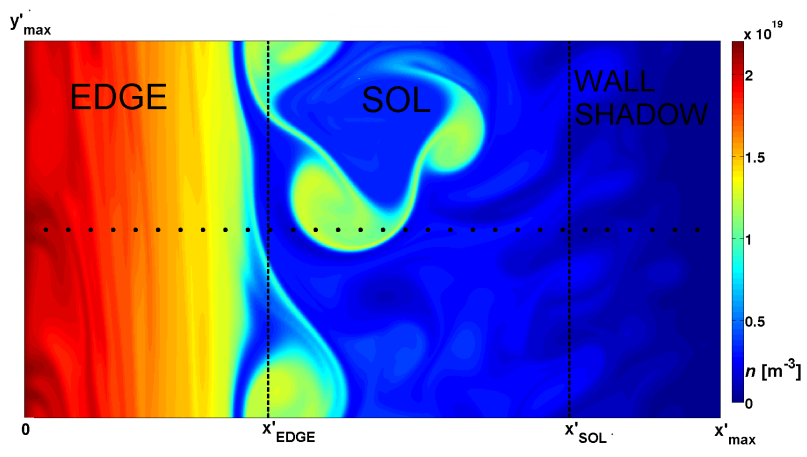

Figure 3. 2D computational domain of ESEL. The black dots represent the location of the numerical probes. The vertical axis represents the poloidal direction and the horizontal axis represents the radial direction. The colour code represents the plasma density. The SOL region width was around $27 \mathrm{~mm}$ for ASDEX Upgrade and around $37 \mathrm{~mm}$ for COMPASS.

plasma and the plasma outside a midplane wall major radius, respectively. The left edge of the EDGE region is impermeable for the plasma. In this region there are no parallel losses (infinite loss times). The ESEL model does not include drift waves or 3D effects that may be important in the EDGE region. Moreover, the EDGE region can be influenced by the inner boundary's conditions. Therefore, only SOL and WALL SHADOW regions should be considered as physically relevant. As the loss times are directly proportional to $L_{\| \mathrm{m}}$, they are finite and long in the SOL, and finite and short in the WALL SHADOW. However, in the WALL SHADOW region, wall effects such as clouds of neutrals are not included. We focus here only on the results in the SOL region.

To model the experimental probes, evenly radially distributed numerical point probes were inserted in the ESEL computational domain. The time data of the fields $n, T_{\mathrm{e}}$ and $\phi$ from numerical probes in certain radial position were processed in the same way as the relevant, experimentally obtained data of $n_{\mathrm{e}}, T_{\mathrm{e}}$ and $\phi_{\mathrm{p}}$, in the time interval corresponding to the same radial position.

\section{Results}

We performed 15 different ESEL simulations using the ASDEX Upgrade plasma parameters and 3 different simulations using COMPASS plasma parameters. The differences in the simulations were in radial boundary conditions (Neumann or Dirichlet) for plasma density, plasma potential, radial electric field and electron temperature, value of the the neoclassical Pfirsch-Schlüter perpendicular collisional diffusion coefficient for particles $D_{\perp \mathrm{n}}^{\prime}$, value of parallel loss time for particles $\tau_{\| \mathrm{n}}^{\prime}$ or in the form of the bracket $\Psi$ in the last term in the equation (9) (where the exponential form is shown).

As the first, we present here outputs of the ESEL model with new sheath dissipation term. We note that the mechanism of the sheath dissipation was con- 


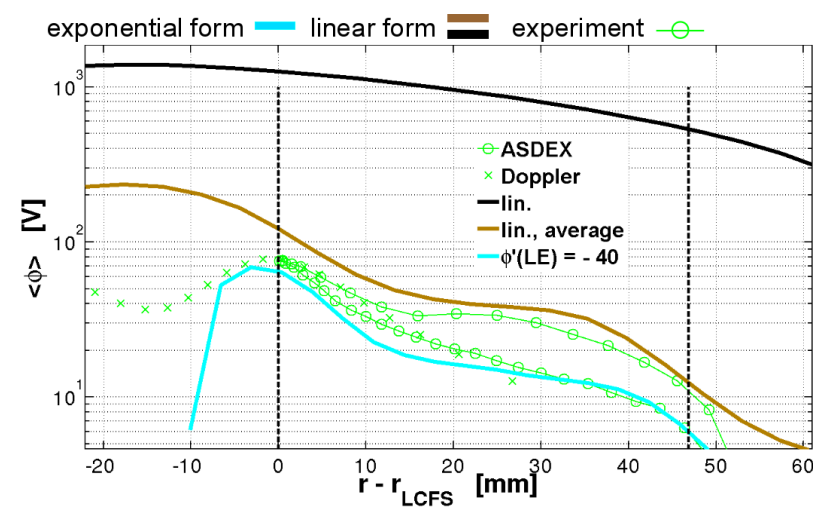

Figure 4. Time-averaged radial profiles of plasma potential. The solid lines represent ESEL data. In the simulation with the exponential form of the $\Psi$ (blue line) the value of electric potential on LE of the computational domain was set as $\phi^{\prime}(\mathrm{LE})=-40$.

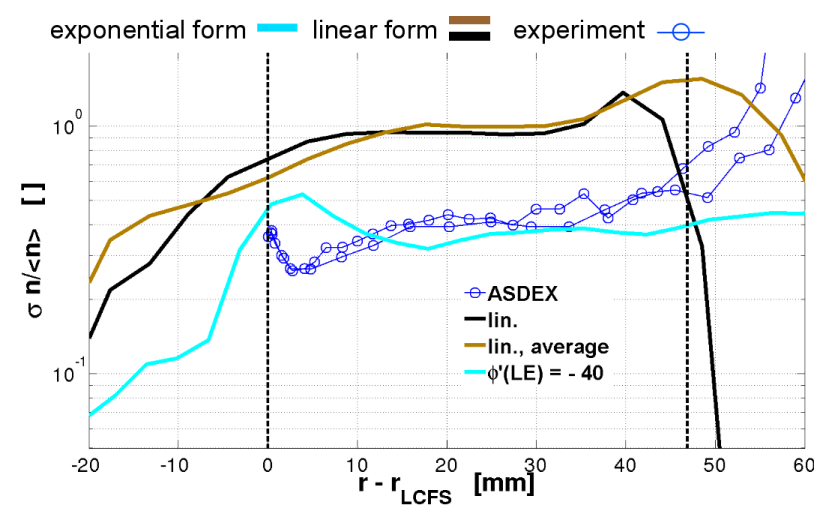

Figure 5. Time-averaged radial profiles of relative plasma density fluctuations. Again, the solid lines represent ESEL data, with the lowest lying, blue line for exponential form of the sheath dissipation term.

sidered inappropriate for highly collisional plasmas 21]. But we may show that its presence in equation (9) via the last term, with $\Psi$ in exponential form, can improve agreement between the model and ASDEX Upgrade experiment. In Figures 4 and 5 the exponential and linear form of the bracket $\Psi$ in the term, is compared for the ASDEX Upgrade tokamak.

The Bohm criterion yields the exponential form of the bracket $\Psi$. The linear form of the $\Psi$ has also been tested mainly for these two reasons: first, we do not know the exact divergence $\nabla \cdot \boldsymbol{J}_{\|}$in the area of the computational domain [26]. Second, the assumption of a sheath-connected plasma implies that it should be collisionless, while the SOL plasma often has significant collisionality. There was a possibility that for non-zero collisionality, the exponential form of the $\Psi$ will not fully apply.

In these two figures we see the agreement for the exponential form in the radial profile of plasma electric potential and relative plasma density fluctuations. The simulation labelled as "lin." has the sheath dissipation term in linear form with $\Psi=\left[\phi^{\prime} / T_{\mathrm{e}}^{\prime}-\alpha\right]$ and the simulations labelled as "lin.average" has the term

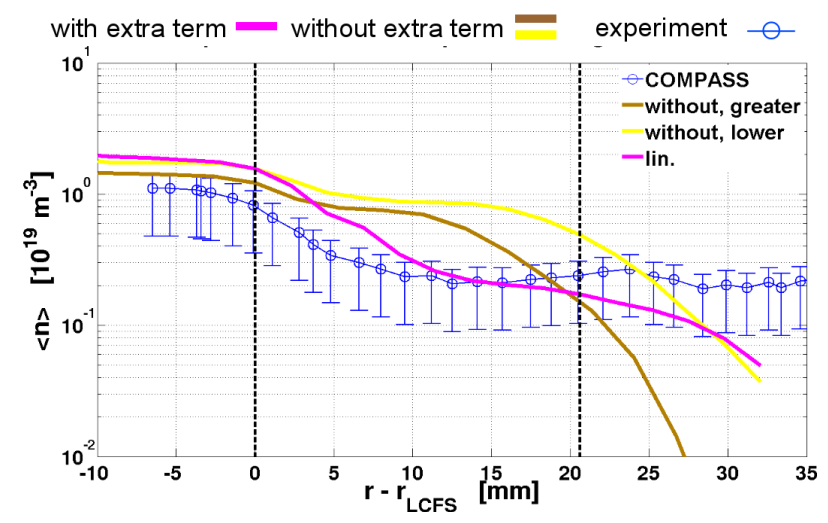

Figure 6. Time-averaged radial profiles of plasma density. The solid lines represent the ESEL data. Sheath dissipation term was used in the simulation represented by the pink solid line.

in the linear 'averaged' form with $\Psi=\left[\left\langle\phi^{\prime}\right\rangle /\left\langle T_{\mathrm{e}}^{\prime}\right\rangle-\alpha\right]$, where \langle\rangle means average over the poloidal direction $y^{\prime}$ of the ESEL computational domain.

In Figure 4 the experimental data of plasma potential labelled as "Doppler" were obtained from the experimental data measured by a Doppler reflectometer. The Doppler reflectometer provides poloidal plasma flow velocity data which we converted to radial electric field data assuming the equality with usual $\boldsymbol{E} \times \boldsymbol{B}$ drift. The assumption that the poloidal motion of the density fluctuations is in fact governed by $\boldsymbol{E} \times \boldsymbol{B}$ drift is valid close to the separatrix [27. In the far SOL, the situation might become more complicated [28. We converted the radial electric field data into plasma potential data by numerical integration with respect to radial distance. The integration constant has been taken into consideration to achieve the best agreement with the probe data labelled as "ASDEX".

In the case of the COMPASS tokamak, we can point to the improvement of the ESEL, using the sheath dissipation term with the bracket $\Psi$ only in linear form. Comparisons of the plasma density radial profile for the simulations without the sheath dissipation term and with the linear sheath dissipation term for the COMPASS tokamak is shown in Figure 6. The words "lower" and "greater" in the labels in this Figure mean only greater and lower $n^{\prime}(L E)$ on the left edge of the ESEL computational domain.

Similar comparisons with and without the sheath dissipation term for the ASDEX Upgrade tokamak can be found in [15] or [5].

In summary, the exponential form of the sheath dissipation term has turned out to be the best form and all simulations for the ASDEX Upgrade presented below were made with the term in this form. In the next section, the consequences of adding this term to the ESEL code are examined in more detail. For clarity, we are presenting only a few representative simulations and experimental data only from one discharge for each tokamak, in all figures in this paper. 


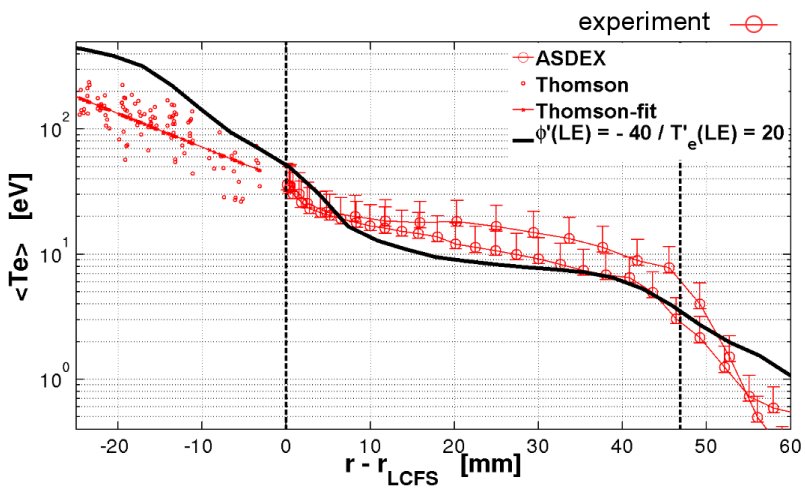

Figure 7. Time-averaged radial profiles of electron temperature (solid line - model, circles - experimental data). The experimental data consist from probe data (right of the LCFS line) and Thomson scattering data (left of the LCFS line - confined plasma).

\subsection{General observations}

For ESEL and the ASDEX Upgrade scrape-off layer profile a good agreement is found for the radial profile of the mean plasma potential (blue solid line in Fig. 4), relative plasma density fluctuations (blue solid line in Fig. 5 and also in the radial profile of mean electron temperature (Fig. 7).

General disagreements for the ASDEX Upgrade tokamak were found, however, in the relative fluctuations of the electron temperature and plasma potential [15] and also in the radial profile of the plasma density (black line in Figure 8). The relative fluctuations are too large compared with the experiment. The gradient of the radial profile of plasma density in the ESEL simulations matching the ASDEX Upgrade plasma is too small compared with the experiment.

In case of the COMPASS tokamak the experimental profile of density has smaller gradient than in the ASDEX Upgrade. The ESEL simulation matches the COMPASS plasma quite well and describes the experimental plasma density radial profile (pink line in Fig. 6. In the case of COMPASS tokamak, there was lower electron collisionality (defined by $\nu_{\mathrm{e}}^{*}=L_{\| \mathrm{m}} / \lambda_{\text {ee }}$ with the electron-electron collisional mean-free path $\lambda_{\text {ee }}$ [21]), than in the case of ASDEX Upgrade tokamak, as we have seen in the ESEL output. In all figures the absolute values (vertical shifting of the graphs) in the mean radial profiles are not so crucial. Gradients of the radial profiles are more important. The values of density and electron temperature at the LCFS are ESEL input. For the COMPASS, in one case (pink line in Fig. 6), the absolute value of the plasma density at the LCFS was set greater intentionally, due to computing speed. For a little greater absolute value of $n_{\mathrm{e}}$ at the LCFS, the diffusion in the computational domain is higher (values of the diffusion coefficients are greater). Also the $\tau_{\| T_{\mathrm{e}}}^{\prime}$ is greater, but the mean radial profile of the electron temperature is not very dependent on it. The values $\tau_{\| \mathrm{n}}^{\prime}, \tau_{\| \Omega}^{\prime}$ do not depend on the density. Overall, the simulation with a little greater value of the $n_{\mathrm{e}}$ at the LCFS is more stable

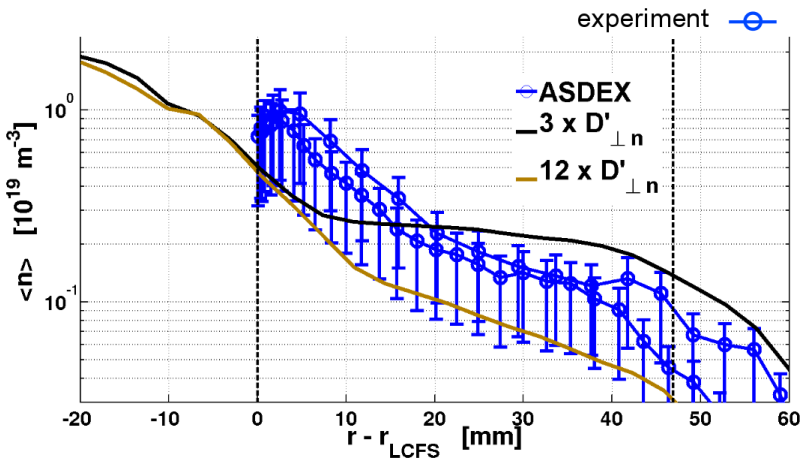

FiguRE 8. Time-averaged radial profiles of plasma density. Two solid lines represent ESEL simulations with theoretical value of the diffusion coefficient for particles multiplied by two different factors.

(the time step of the simulation can be longer) due to dissipation of the smallest structures (because of higher diffusion); while a change in the dynamics of the turbulent structures, that exist at larger spatial scales, is negligible.

We have tried to improve the general mismatches for the ASDEX Upgrade tokamak by changing the ESEL parameters and some of the results are presented in the next section.

\subsection{Parameter Dependence}

We changed the parameters $D_{\perp \mathrm{n}}^{\prime}$ and $\tau_{\| \mathrm{n}}^{\prime}$ and observed their impact on the ESEL dynamics. Our main purpose was to increase the gradient of the radial profile of plasma density in ESEL simulations matching the ASDEX Upgrade plasma. This increase was achieved by an increase in $D_{\perp \text { n }}^{\prime}$ as we can see in Fig. 8 .

There is a rather large uncertainty in the value of theoretically derived coefficient $D_{\perp \mathrm{n}}^{\prime}$. First, it is only an effective radial value obtained by flux surface averaging. Second, it assumes the existence of closed flux surfaces that is, indeed, not present in SOL. A rigorous treatment of neoclassical transport on open flux surfaces has yet to be performed, [21]. Therefore we have tested the consequences of increasing the theoretical value of $D_{\perp n}^{\prime}$ by a factor in the range of $1.5-12$ which corresponds to the absolute values in the range of around $8.9 \times 10^{-4}-7.1 \times 10^{-3}$.

For $D_{\perp \mathrm{n}}^{\prime}$ multiplied by factor 12 the gradient of the plasma density radial profile is in a good concurrence with the experiment (Fig. 8). The simulation, however, represents a plasma with almost no blobs crossing LCFS and consequently almost no density fluctuations close to the LCFS (see Fig. 9), therefore such a large diffusion coefficient seems to be incorrect.

We also tested whether an increase in parallel loss (along the magnetic field) of particles may increase the gradient of the plasma density radial profile.

And we found a decrease in $\tau_{\| \mathrm{n}}^{\prime}$ (within the theoretical uncertainty) doesn't increase the gradient significantly and it also causes an increase of relative density and temperature fluctuations well above the 


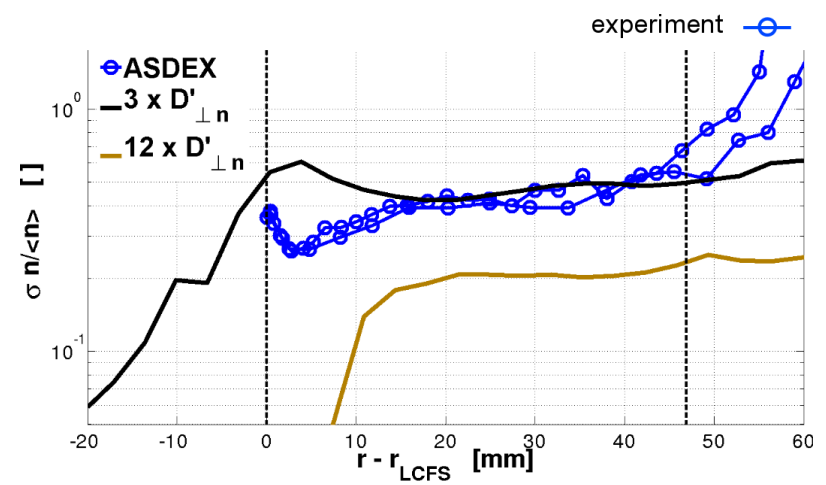

Figure 9. Time-averaged radial profiles of relative plasma density fluctuations. The solid lines represent ESEL data with theoretical value of the diffusion coefficient multiplied by a factor of 3 and 12 .

experimentally observed level [15]. Therefore, the increase in parallel losses of particles characterized by parallel particle density loss time is not a solution to have a steeper radial density profile.

\section{Conclusions}

We compared 15 ESEL simulation runs with experimental data from the ASDEX Upgrade \#24349 and 3 simulations with experimental data from the COMPASS tokamak. In the standard ESEL code we have included a sheath dissipation term in the vorticity equation to investigate if we can observe an improved agreement with experimental observations for the profiles of density, electric potential and electron temperature. We considered exponential and linear form of the term.

We observed a strong effect using the sheath dissipation term which generally makes a better match to the experimental observations of ASDEX Upgrade but, e.g., the gradient of radial profile of the plasma density is too small compared to the experiment.

We also tested an increase in the perpendicular collisional diffusion coefficient $D_{\perp \mathrm{n}}^{\prime}$ and the parallel losses of density characterized by parallel particle density loss time $\tau_{\| \mathrm{n}}^{\prime}$ to obtain a better agreement with the experimental observations, but without success. The summary of which profiles obtained from ESEL match the ASDEX Upgrade observation is shown in Table 1 .

For the simulations using COMPASS parameters, we observe a better agreement with the experiment for the density gradient but only 3 simulations were studied from which two simulations do not have the sheath dissipation term, and one simulation has only a linear form of the sheath dissipation term.

Generally, we conclude that the present ESEL model cannot fully simulate the experimental observation from ASDEX Upgrade. There may be other dissipative processes, such as drift waves [29] which are not included in the ESEL model, and which dissipate the turbulence structures, resulting in a steeper experimental radial density profile. An extension of

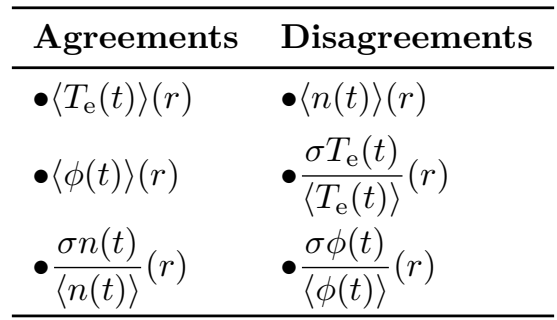

TABLE 1. General conclusions about the model ESEL matching the ASDEX Upgrade tokamak plasma.

the ESEL model from 2D to 3D to fully resolve the parallel dynamics and the coupling from the plasma to the sheath is necessary to improve agreements.

\section{ACKNOWLEDGEMENTS}

This research has been supported by the Czech Science Foundation project P205/12/2327 and MSMT Project No. LM2011021.

Access to computing and storage facilities owned by parties and projects contributing to the National Grid Infrastructure MetaCentrum, provided under the programme "Projects of Large Infrastructure for Research, Development, and Innovations" (LM2010005), is appreciated.

\section{REFERENCES}

[1] ANTAR, Y., G.et al. On the scaling of avaloids and turbulence with the average density approaching the density limit. Physics of Plasmas 12, 082503 (2005). DOI:10.1063/1.19535592

[2] GARCIA, O., E. et al. Turbulence and intermittent transport at the boundary of magnetized plasmas. Physics of Plasmas 12, 062309, (2005). DOI:10.1063/1.1925617

[3] GARCIA, O., E. et al. Interchange turbulence in the TCV scrape-off layer. Plasma Phys. Control. Fusion 48, L1-L10, (2006). DOI:10.1088/0741-3335/48/1/L01

[4] GARCIA, O., E. et al. Turbulence simulations of blob formation and radial propagation in toroidally magnetized plasmas. Phys. Scr. T112, 89-103, (2006). DOI:10.1088/0031-8949/2006/T122/013

[5] VONDRÁČEK, P.. Study of edge plasma physics of tokamak COMPASS by means of two reciprocating probes. Master thesis, CTU in Prague, Czech Republic, 2012

[6] HERRMANN, A. , GRUBER, O. ASDEX

Upgrade-Introduction and Overview. Fusion Science and Technology, Vol. 44, No. 3, 569 - 577, (2003).

[7] PÁNEK, R. et al. Reinstallation of the COMPASS-D tokamak in IPP ASCR. Czechoslovak Journal of Physics, Vol. 56, issue 2, pp. B125 - B137, (2006). DOI:10.1007/s10582-006-0188-1

[8] NAULIN, V. et al. Turbulence modeling of JET SOL Plasma. In Proceedings of the 21st IAEA Fusion Energy Conference, (Chengdu, China, 2006), TH/P6-22. online at http://www.iop.org/Jet/fulltext/EFDC060519.pdf [2015-03-01] 
[9] VERGOTE, M., et al. Discussion of SOL turbulence properties in TEXTOR by means of ESEL simulations . In 38th EPS Conference on Plasma Physics, 2011, P5.068. online at http://ocs.ciemat.es/EPS2011PAP/pdf/P5.068.pdf [2015-03-01]

[10] MILITELLO, F.,et al. Simulations of edge and scrape off layer turbulence in mega ampere spherical tokamak plasmas. Plasma Phys. Control. Fusion 54, No.9, 095011, 2012 DOI:10.1088/0741-3335/54/9/095011

[11] GARCIA, O., E.,et al. Fluctuations and transport in the TCV scrape-off layer. Nucl. Fusion 47, No. 7, p. 667-676, (2007) DOI:10.1088/0029-5515/47/7/017

[12] GARCIA, O., E.,et al. Collisionality dependent transport in TCV SOL plasmas. Plasma Phys. Control. Fusion 49, (2007) B47-B57. DOI:10.1088/0741-3335/49/12B/S03

[13] GARCIA, O., E.,et al. Turbulent transport in the TCV SOL. Journal of Nuclear Materials 363-365, (2007) 575. DOI:10.1016/j.jnucmat.2006.12.063

[14] ADÁMEK, J. et al. A novel approach to direct measurement of the plasma potential. Czechoslovak Journal of Physics, Vol. 54, pp C95-C99, (2004). DOI:10.1007/BF03166386

[15] ONDÁČ, P.. Study of tokamak plasma turbulence by means of reciprocating probes. Master thesis, Charles University in Prague, Czech Republic, 2014.

[16] HORACEK, J. et al. Interpretation of fast measurements of plasma potential, temperature and density in SOL of ASDEX Upgrade. Nucl. Fusion 50, No.10, 105001,(2010). DOI:10.1088/0029-5515/50/10/105001

[17] ADÁMEK, J. et al. Ball-Pen Probe Measurements in L-Mode and H-Mode on ASDEX Upgrade. Contrib. Plasma Phys. 50, No. 9, 843-859, (2010). DOI:10.1002/ctpp.201010145

[18] ADÁMEK, J. et al. Direct plasma potential measurements by ball-pen probe and self-emitting Langmuir probe on COMPASS and ASDEX Upgrade. Contrib. Plasma Phys. 54, Issue 3, pp. 279-284, (2014). DOI:10.1002/ctpp.201410072
[19] ADÁMEK, J. et al. Fast ion temperature measurements using ball-pen probes in the SOL of ASDEX Upgrade during L-mode. $38^{\text {th }}$ EPS Conference on Plasma Physics, Vol. 35G, P1.059 (2011).

[20] KOČAN, M. et al. Edge ion-to-electron temperature ratio in the Tore Supra tokamak. Plasma Phys. Control. Fusion 50, No. 12, 125009, (2008). DOI:10.1088/0741-3335/50/12/125009

[21] FUNDAMENSKI, W. et al. Dissipative processes in interchange driven scrape-off layer turbulence. Nucl.

Fusion 47, 417-433 (2007). DOI:10.1088/0029-5515/47/5/006

[22] GARCIA, O., E. et al. Radial interchange motions of plasma filaments. Physics of Plasmas 13, 082309 (2006). DOI:10.1063/1.2336422

[23] SARAZIN, Y. , Ghendrih, Ph. Intermittent particle transport in two-dimensional edge turbulence. Physics of Plasmas 5, 4214 (1998). DOI:10.1063/1.873157

[24] BISAI, N. et al. Simulation of plasma transport by coherent structures in scrape-off-layer tokamak plasmas. Physics of Plasmas 11, 4018 (2004). DOI:10.1063/1.1771658

[25] GARCIA, O., E. et al. Turbulence and intermittent transport at the boundary of magnetized plasmas. Physics of Plasmas 12, 062309 (2005). DOI:10.1063/1.1925617

[26] KRASHENINNIKOV, S., I. et al. Recent theoretical progress in understanding coherent structures in edge and SOL turbulence. J. Plasma Physics 74, part 5, pp. 679-717 (2008). DOI:10.1017/S0022377807006940

[27] MüLLER, H., W. et al. Latest investigations on fluctuations, ELM filaments and turbulent transport in the SOL of ASDEX Upgrade. Nucl. Fusion 51, 073023, 11pp (2011). DOI:10.1088/0029-5515/51/7/073023

[28] MüLLER, H., W. et al. Characterization of Scrape-Off Layer Turbulence Changes Induced by a NonAxisymmetric Magnetic Perturbation in an ASDEX Upgrade Low Density L-Mode. Contrib. Plasma Phys. 54, No.3, 261-266 (2014). DOI:10.1002/ctpp.201410079

[29] ANGUS, J., R. et al. Effect of Drift Waves on Plasma Blob Dynamics. Phys. Rev. Lett. 108, 215002 (2012). DOI:10.1103/PhysRevLett.108.215002 